\title{
Solar System Science with Space Telescopes
}

White Paper for the Planetary Science Decadal Survey

Principal Author

Roser Juanola-Parramon

NASA Goddard Space Flight Center/ University of Maryland Baltimore County roser.juanola@nasa.gov

Phone: 301-614-5837

\section{Co-authors}

Heidi B. Hammel (AURA)

Giada Arney (NASA GSFC)
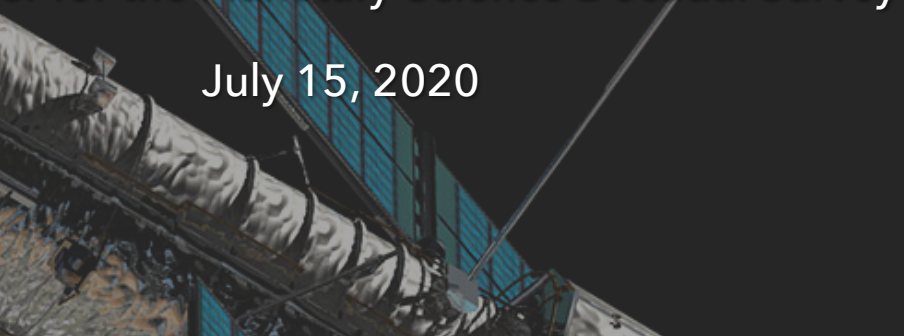

Aki Roberge (NASA GSFC)

Geronimo Villanueva (NASA GSFC)

Walter Harris ( $U$ of Arizona, LPL)

Britney Schmidt (Georgia Tech)

Kelsi Singer (SwRI)

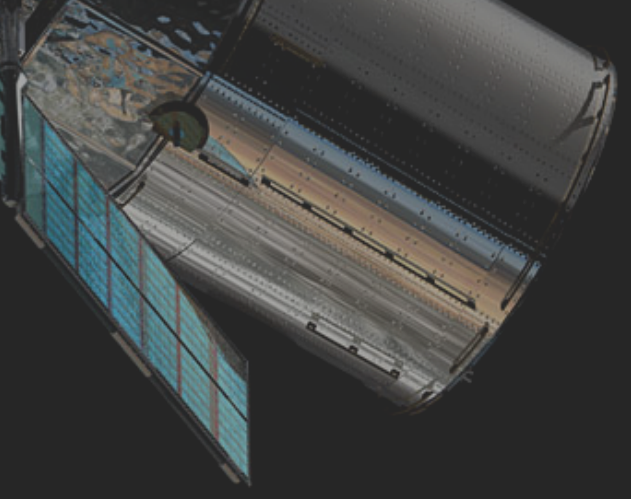




\section{Abstract}

Building on the legacy of the Hubble Space Telescope and other space telescopes, a large multi-wavelength space observatory would enable transformative advances in a broad range of planetary science topics. Remote sensing observations could provide longduration monitoring, as well as observations of bodies that will not be visited by orbiter or flyby spacecraft in the foreseeable future. Access to ultraviolet wavelengths not observable from ground-based telescopes is also a key advantage in several areas (e.g., aurorae on both planets and moons). Here we highlight several representative areas of Solar System science that would greatly benefit from high resolution UV/optical/NIR imaging, focusing specifically on Venus, Jupiter, Neptune, dwarf planets, and Kuiper Belt Objects.

\section{Context for Solar System Studies with Space Telescopes}

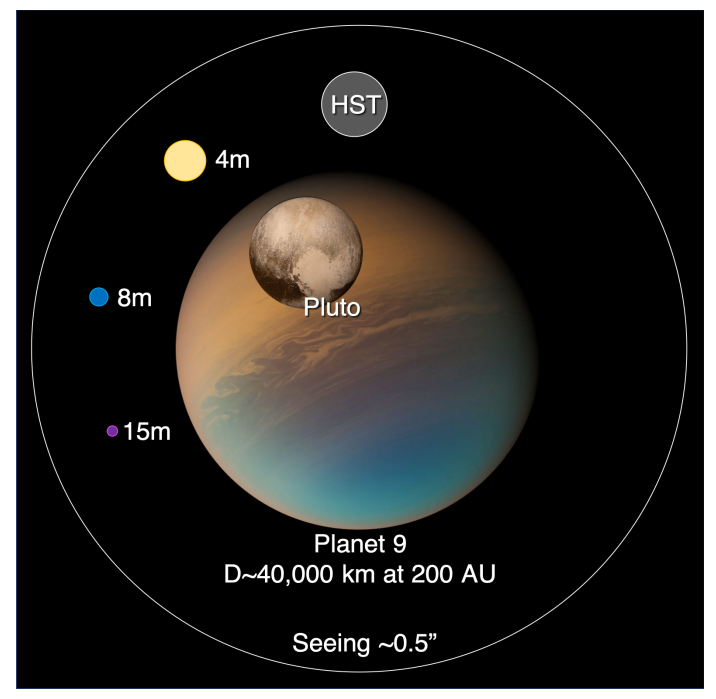

This image illustrates angular resolutions corresponding to 0.5 arcsec seeing (white ring) and space telescopes with apertures ranging from 2.4-m (Hubble Space Telescope) to 15-m. With increased angular resolution and sensitivity, space telescopes can resolve and monitor the farthest objects in our Solar System like the suspected "Planet 9" (Brown and Batygin 2016).

The Hubble Space Telescope has proven to be a valuable tool for Solar System studies (e.g., see review by James and Lee 1999). From bodies small (asteroids, comet nuclei) to large (Jupiter, Saturn), Hubble observations complemented planetary science in situ missions, and in some cases enabled new goals or extended missions (e.g., Hubble's discovery of 2014MU69, the second target of the New Horizons spacecraft, through a dedicated search). The power of astrophysics missions for Solar System exploration was acknowledged by NASA for the James Webb Space Telescope (see Milam et al. 2016 and references therein): JWST Solar System observations are included in both Guaranteed Time observations ${ }^{1}$ and Early Release Science observations ${ }^{2}$.

In the same vein, future space telescopes will also be a powerful complement to future in situ planetary spacecraft. This white paper is not meant to be a comprehensive review of Solar System science needs that could be supplied by remote sensing with

\footnotetext{
1 https://jwst.stsci.edu/observing-programs/approved-gto-programs

2 https://jwst.stsci.edu/observing-programs/approved-ers-programs
} 
future space telescopes. Rather, it briefly illustrates a range of high-quality investigations that next-generation large space telescopes could accomplish.

\section{Imaging the Solar System with Next-Generation Space Telescopes}

\section{Venus}

Venus is our nearest planetary neighbor, yet many questions about this complex veiled world remain unanswered. Recent orbiters have revealed a highly dynamic atmosphere with recurring patterns such as a global-scale cloud-top wave feature observed by JAXA's Akatsuki's Longwave Infrared Camera (Fukuhara et al. 2017). This feature appears to be tied to surface topography and the time of day, necessitating long time-baseline observations to study it given Venus' 116 day-long sidereal day. Space-based observations could image cloud top features in exquisite detail (Fig. 1). A 15-m telescope could obtain resolution as high as $6 \mathrm{~km}$ per resolution element at $400 \mathrm{~nm}$ and $18 \mathrm{~km}$ at $1200 \mathrm{~nm}$, comparable to flyby and orbiter facilities.

In addition, a long-standing mystery of the Venus atmosphere is the identity of its "unknown UV absorber", which, together with $\mathrm{SO}_{2}$, is responsible for absorbing more than half of the solar radiation incident on Venus. Observations at UV wavelengths may shed light on the identity of this UV absorber. For instance, observations of cloud patterns driven by zonal winds at UV wavelengths could indicate the absorber's altitude (e.g. Horinouchi et al. 2018).

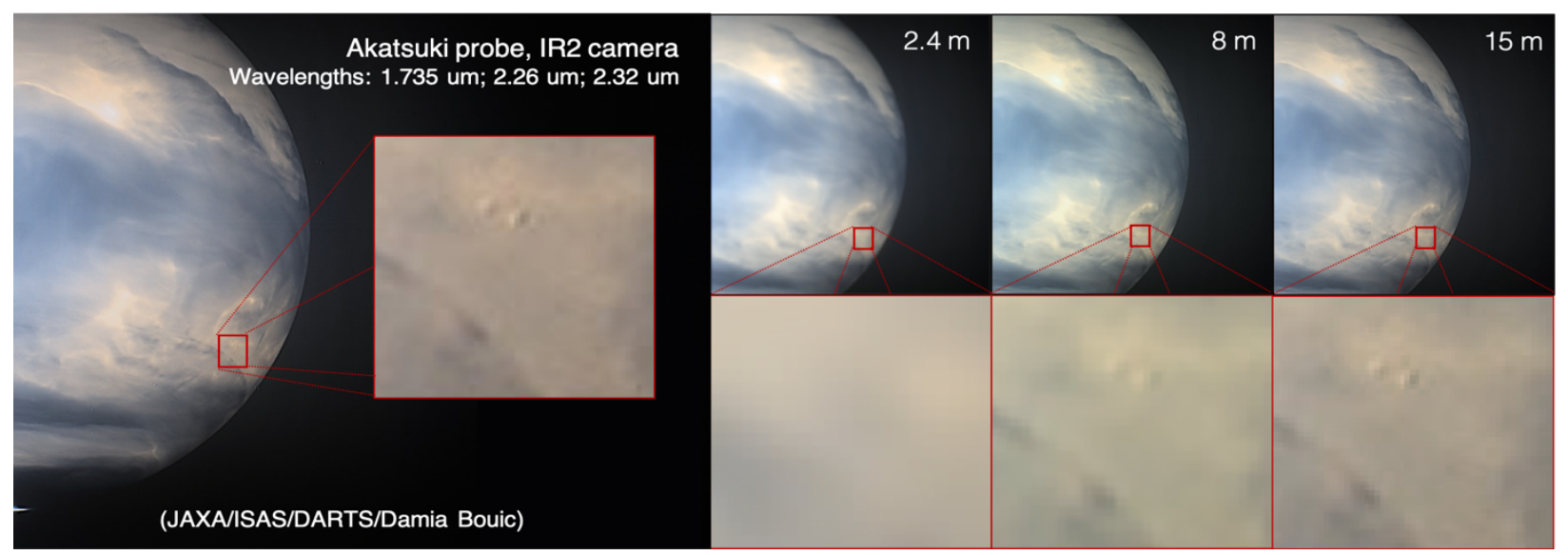

Figure 1: Venus. Currently, there is no planned NASA mission to Venus. Periodic monitoring of Venus over long-time baselines can reveal variations in atmospheric species that may constrain theories of dynamical, chemical, and geophysical processes. This can be accomplished with a space observatory that can point to a minimum solar elongation angle of $<45^{\circ}$, which is small enough to enable observations of Venus near maximum elongation. 


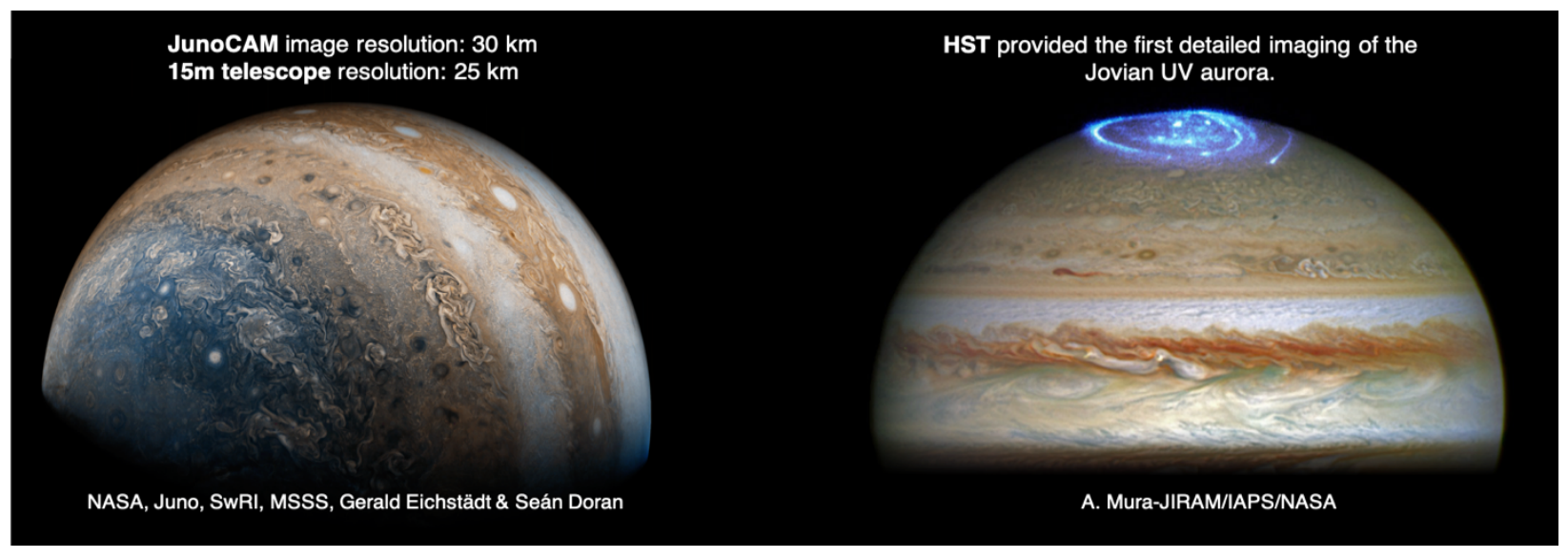

Figure 2: Jupiter. Left: JunoCam image of Jupiter's southern hemisphere, with spatial resolution comparable to that of a 15-m diameter optical space telescope. Right: Ultraviolet Ly-alpha emission in the polar auroral region obtained with HST STIS. A large aperture space observatory provides not only increased spatial resolution, but also increased sensitivity. With increased sensitivity comes shorter exposures, reducing rotational blurring of features and permitting high-fidelity studies of the dynamics of Jovian aurora.

\section{Jupiter System}

The Juno spacecraft has been returning stunning images of Jupiter since July 2016 (Fig. 2, left), and will continue until a planned de-orbit in July 2021. To deliver images with resolution comparable to Juno images ( $25 \mathrm{~km}$ at $500 \mathrm{~nm}$ ), a space telescope near Earth would need a diameter of about 15 meters. The particular advantage of a spacebased platform is the ability to provide diffraction-limited imaging over wide fields of view, which will be a challenge for future ground-based telescopes equipped with extreme adaptive optics, especially at optical wavelengths. A telescope located in the ecliptic plane will not be able to observe Jupiter's polar regions as well as Juno can, but will provide longitudinal coverage of the entire planet.

Just as Hubble observations of Jovian and Saturnian aurorae (Fig. 2, right) complemented missions such as Juno and Cassini respectively, a future UV space telescope would complement giant planet missions in future decades. Furthermore, with extreme angular resolution and sensitivity, such an observatory could provide repeat imaging and spectroscopy of icy moons like Europa over long timescales. This would allow time variability studies of geysers, atmospheric chemistry, and surface composition. Interactions between the magnetospheres of Ganymede and Jupiter could be studied with the $\sim 10 \mathrm{~km}$ resolution of a 15-m-class, UV-capable telescope.

\section{Neptune}

No spacecraft has visited Uranus and Neptune in over three decades. While advances in outer planet science have been made with Hubble, the Keck 10-m telescopes, and other ground-based facilities, the extreme distances of these planets hamper high- 


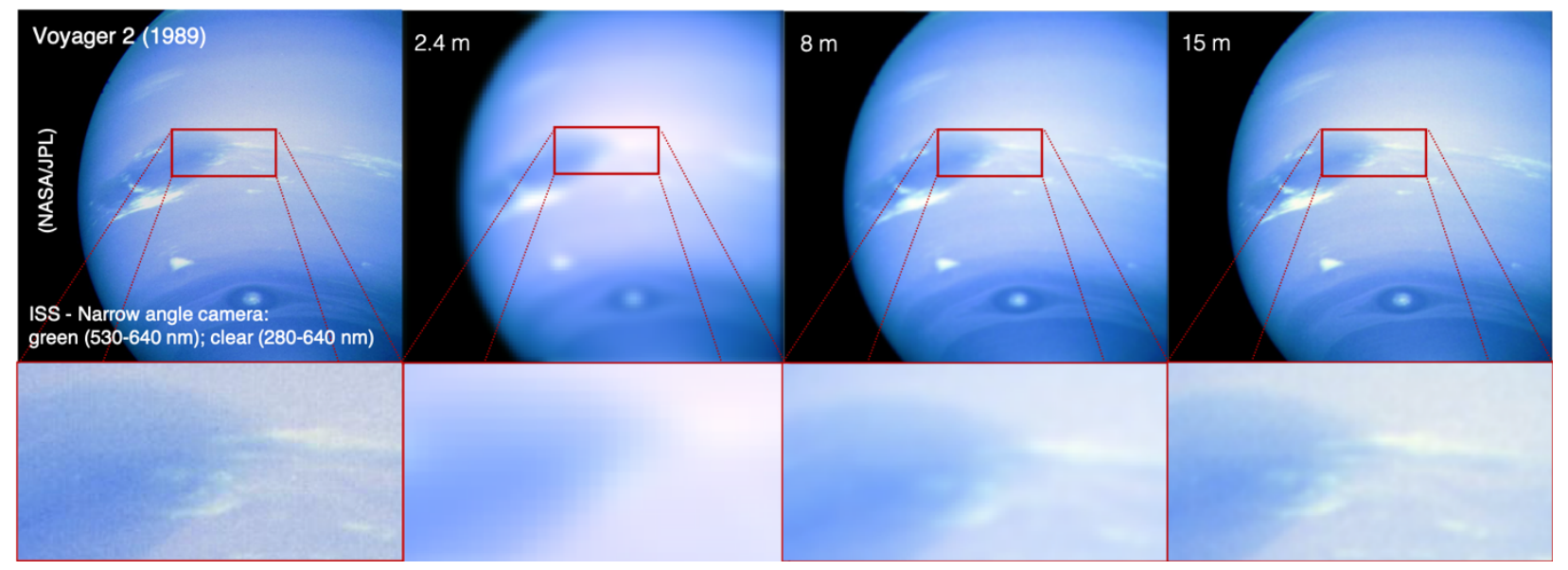

Figure 3: Neptune. Far-left: Image of Neptune reconstructed from two Voyager 2 narrow-angle camera images. Simulated Neptune images as seen with 2.4-m, 8-m and 15-m telescopes, demonstrating that spatial resolution comparable to that of the Voyager 2 spacecraft is achieved at $\sim 15 \mathrm{~m}$.

quality imaging of their atmospheres. As an example, Hubble observations of Neptune have captured the appearance and disappearance of multiple dark spots on Neptune, yet effectively nothing is known about what triggers these features, nor what causes their dissipation. A 15-m aperture space telescope could capture images of Neptune (Fig. 3) that rival the quality of Voyager 2 flyby images. Uranus' apparent disk subtends 3.8" compared with Neptune's tiny 2.3" disk; thus, Uranus images will be concomitantly better. Such observations of ice giants will permit major advances in our understanding of their atmospheric dynamics.

\section{Characterization of Dwarf Planets \& Small Bodies}

HST revolutionized the study of asteroids primarily through high resolution imaging, transforming them from points of light into worlds of their own (e.g. Thomas et al. 2005, Li et al. 2006, Schmidt et al. 2009). In particular, these high resolution images (between $\sim 25 \mathrm{~km} /$ pixel for ACS and $\sim 40 \mathrm{~km} /$ pixel for WFPC2 \& 3) allowed the detailed shapes of these bodies to be measured, which discovered major impact basins sculpting the surfaces of Vesta and Pallas, and the hydrostatic state of Ceres that, along with its low density, predicted its ice rich outer shell that is now confirmed by the Dawn mission (Thomas et al. 2005, McCord and Sotin 2005, Prettyman et al. 2016). A 15-m space telescope could accomplish much better than this level of science for the entire asteroid belt, achieving kilometer scale resolution throughout the main belt, and $\sim 10$ $\mathrm{km}$ for Trojans. Combining high resolution shape models, detailed surface maps, and spectroscopy, this extends nearly Discovery mission-scale science to the whole of the asteroid belt, providing a chance to compare these bodies as planets and as survivors of the chaos of the early solar system. 


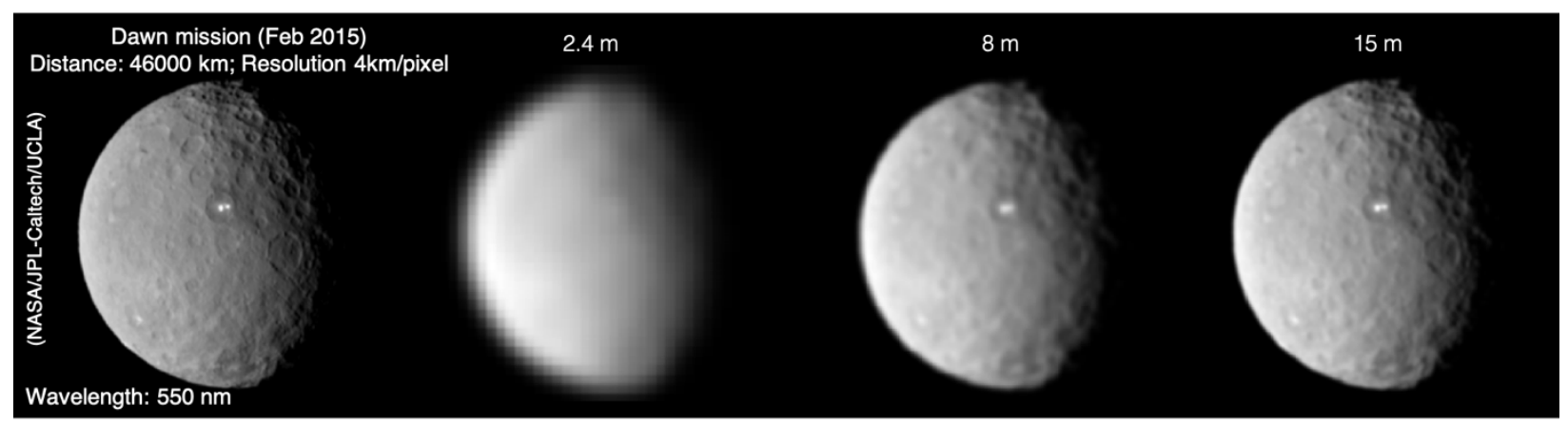

Figure 4: Ceres. Since Dawn arrived in orbit at Ceres in March 2015, scientists have located more than 300 bright areas on Ceres - demonstrating that regional and local variations occur on large asteroids. Bright spots reveal evidence of Ceres' past subsurface ocean and indicate that Ceres is surprisingly active.

Large space telescopes would have the ability to study the geophysical evolution of dwarf planets and small bodies alike. While Ceres (Fig. 4) has already been imaged at high resolution, HST studies and the Dawn mission have confirmed that its surface changes albedo due to impacts and mass wasting of its ice rich crust (e.g. Thomas et al. 2005) such that changes on Ceres surface would be visible to a space-based telescope with access to the NUV. Moreover, debate continues as to whether Ceres currently outgasses or whether sublimation occurs in bursts due to solar activity or surface disruptions (e.g. Kuuppers et al. 2013, Landis et al. 2018), which a UV-enabled space telescope could contribute higher signal observations to survey for variation.

For Pluto (Fig. 5), Triton (which began its life as a dwarf planet and TNO), and similar bodies in the trans-Neptunian region, a large space telescope would transform monitoring of global scale activity. With surface temperatures near the triple point of methane and nitrogen, surface-atmosphere transport and interactions lead to dramatic shifts in the surface color, composition, and distribution of materials that were

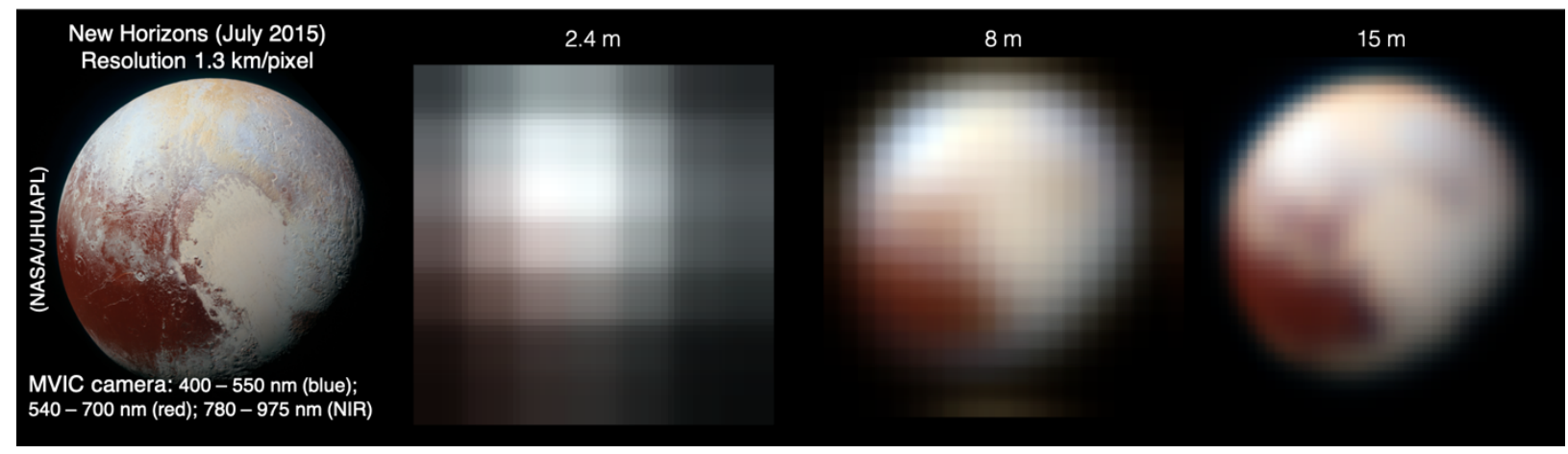

Figure 5. Pluto. With high spatial resolution and spectroscopy in the $0.5-3.5 \mathrm{~nm}$ range, a large space observatory can provide spectral characterization ranging from high fidelity spatial, diurnal, and seasonal coverage of larger Dwarf Planets to more complete identification of surface composition on smaller bodies. 
observable even with HST (Bauer et al. 2006). These changes are seasonal (e.g. Hansen et al. 1992) and sporadic (e.g. Buratti et al. 1994) possibly due to active cryovolcanism. At $<50-100 \mathrm{~km}$ resolution from Neptune's orbit through the Kuiper Belt, surface maps derived from a 15-m space telescope can monitor seasonal and other changes of the surfaces and atmospheres of these bodies.

For Pluto, this represents the unique chance to tie high resolution data of the system at one instance to its behavior over a significant portion of its seasons, providing new information on its own geologic evolution. Connecting our knowledge of Pluto and Triton across the Kuiper Belt will transform how we understand dwarf planets and KBOs and their changes with time.

\section{Kuiper Belt Object (KBO) survey}

Icy bodies in the outer solar system are remnants of the processes that led to the formation and current distribution of the giant planets. It is only over the last 25 years (Jewitt \& Luu 1993) that we have begun to map the distribution of Trans-Neptunian Objects in the Kuiper-Edgeworth Belt (KEB), the inner of two icy body reservoirs. The major factor limiting further study of icy planetesimals is the detection efficiency for small bodies. These objects are only visible due to reflected sunlight that experiences inverse square dilution in both the outbound and reflected directions, resulting in an $\mathrm{R}^{-4}$ brightness function with heliocentric distance. Dynamical models identifying the KEB as the source of short-period comets (SPCs) and Centaurs require that $\mathrm{KBOs}<10$ $\mathrm{km}$ in diameter must be extremely common. Hubble, currently the most sensitive telescope for detection of small KBOs, has detected a $30-\mathrm{km}$ diameter object in a circular orbit at $43 \mathrm{AU}$ in reflected sunlight (2014 MU69; Buie et al. 2018; Porter et al. 2019). Extending the detection threshold down to the 1-10 km sizes typical of SPCs will require an increase in sensitivity of about a factor of 4 to 400 over HST.

\section{Beyond Imaging - Spectroscopy of Solar System Objects}

The spectroscopic capability of the next generation of space observatories will yield significant contributions to Solar System science. It will be especially fruitful for small bodies, where sheer numbers preclude the possibility of sending spacecraft to even a moderate number of representative samples. Asteroids, KBOs, comets, Centaurs, and NEOs will all be potential targets for spectroscopic characterization. The addition of spectroscopic measurements to high resolution imaging, particularly if that spectroscopy can be spatially resolved, presents a chance to dramatically change our understanding of these bodies individually as planets and as classes of objects that inform the grand scale architecture of solar systems far and near. 


\section{Summary}

A large space observatory will provide unprecedented Solar System science and would nicely complement future orbiters and landers. Dedicated planetary missions will uniquely permit in situ measurements of optically inactive noble gases, gravity data, as well as particle and field measurements. Yet, as history has shown from our experience with Hubble, Solar System exploration with a powerful and general observatory greatly complements and advances in situ planetary missions.

\section{References}

Bauer, J. M., et al. (2010). Direct detection of seasonal changes on Triton with Hubble Space Telescope. The Astrophysical Journal Letters, 723

Brown, M. E. \& Batygin, K. (2016). Observational constraints on the orbit and location of Planet Nine in the outer solar system. The Astrophysical Journal Letters, 824

Buie, M., et al. (2018, October). Pre-encounter update on (486958) 2014MU69 and occultation results from 2017 and 2018. In AAS/Division for Planetary Sciences Meeting Abstracts (Vol. 50).

Buratti, B. J., Goguen, J. D., Gibson, J., \& Mosher, J. (1994). Historical photometric evidence for volatile migration on Triton. Icarus, 110

Fukuhara, T., et al. (2017). Large stationary gravity wave in the atmosphere of Venus. Nature Geoscience, 10

Hansen, C. J. \& Paige, D. A. (1992). A thermal model for the seasonal nitrogen cycle on Triton. Icarus, 99

Horinouchi, T., et al. (2018). Mean winds at the cloud top of Venus obtained from two-wavelength UV imaging by Akatsuki. Earth, Planets and Space, 70

James, P. B. \& Lee, S. W. (1999). Hubble Space Telescope Observations of Planets and Satellites. Ann. Rev. Earth and Planetary Sciences $\mathbf{2 7}$

Jewitt, D. \& Luu, J. (1993). Discovery of the candidate Kuiper belt object 1992 QB1. Nature, 362

Küppers, M., et al. (2014). Localized sources of water vapour on the dwarf planet (1) Ceres. Nature, 505

Landis, M. E., et al. (2017). Conditions for sublimating water ice to supply Ceres' exosphere. Journal of Geophysical Research: Planets, 122

Li, J. Y., McFadden, et al. (2010). Photometric mapping of Asteroid (4) Vesta's southern hemisphere with Hubble Space Telescope. Icarus, 208

McCord, T. B. \& Sotin, C. (2005). Ceres: Evolution and current state. Journal of Geophysical Research: Planets, 110

Milam, S. et al. (2016). Special Issue: Innovative Solar System Science with the James Webb Space Telescope, Pub. Astron. Soc. Pac. 128

Porter, S., et al. (2019). VizieR Online Data Catalog: Astrometry of (486958) 2014 MU69 with HST (Porter+). VizieR Online Data Catalog, 515

Prettyman, T. H., et al. (2017). Extensive water ice within Ceres' aqueously altered regolith: Evidence from nuclear spectroscopy. Science, $\mathbf{3 5 5}$

Schmidt, B. E., et al. (2009). The shape and surface variation of 2 Pallas from the Hubble Space Telescope. Science, 326

Thomas, P. C., et al. (2005). Differentiation of the asteroid Ceres as revealed by its shape. Nature, 437 